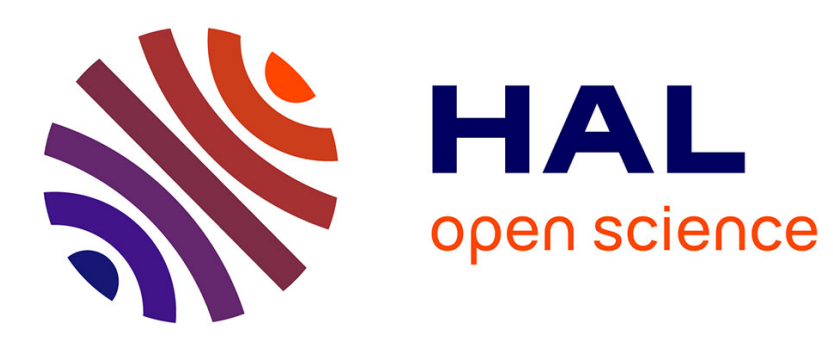

\title{
Codes et codages professionnels dans les mondes de l'information
}

Gilles Bastin

\section{To cite this version:}

Gilles Bastin. Codes et codages professionnels dans les mondes de l'information. Réseaux: communication, technologie, société, 2009, 157-158 (5-6), pp.191-211. 10.3917/res.157-158.0191 . halshs00450206

\section{HAL Id: halshs-00450206 https://shs.hal.science/halshs-00450206}

Submitted on 19 Dec 2019

HAL is a multi-disciplinary open access archive for the deposit and dissemination of scientific research documents, whether they are published or not. The documents may come from teaching and research institutions in France or abroad, or from public or private research centers.
L'archive ouverte pluridisciplinaire HAL, est destinée au dépôt et à la diffusion de documents scientifiques de niveau recherche, publiés ou non, émanant des établissements d'enseignement et de recherche français ou étrangers, des laboratoires publics ou privés. 
Gilles BASTIN

Paru dans Réseaux, 157-158, 2009, p. 192-211

[hal-00450206] | [http://www.cairn.info/revue-reseaux-2009-5-page-191.htm]

DOI: $10.3917 /$ res.157-158.0191

Le journalisme est-il une profession régulée ? Dans les sociétés contemporaines, la question est régulièrement posée dans l'espace public, périodiquement agité par des interrogations sur la « qualité »du travail des journalistes. Elle est aussi posée, de façon récurrente, dans la sociologie du journalisme. Celle-ci, peut-être à cause de ces débats publics sur la fonction des journalistes dans la société, a en effet longtemps examiné le journalisme en se référant au modèle des professions régulées, comme la médecine ou le droit. Le journalisme a ainsi été décrit comme un métier en voie de " professionnalisation » pour reprendre le raisonnement en termes d'échelles et de progression linéaire typique du modèle développé par $\mathrm{H}$. Wilensky dans les années $1960^{1}$.

Sans entrer ici dans le détail d'une question complexe, on peut noter que malgré le travail de professionnalisation et de codification entrepris en France par les associations représentatives de journalistes depuis les années $1920^{2}$, la régulation de l'activité des professionnels de l'information reste extrêmement faible. Dans le domaine de la formation et de l'accès au métier (Sales, 1998 ; Marchetti, 2003 ; Pelissier \& Ruellan, 2003)3, du maintien d'une cohérence identitaire professionnelle (Charon, 1992 ; Leteinturier, 2003) ou encore du contrôle des carrières professionnelles des membres de la profession (Direction du développement des médias \& CRAP, 2001), il est difficile d'observer l'existence d'un « code

\footnotetext{
1 Le modèle de Wilensky considère le processus de professionnalisation comme un développe- ment linéaire conduisant les métiers - certains d'entre eux du moins - à devenir des professions régulées. Les grandes étapes de ce processus sont les suivantes : le passage à un exercice à plein temps, l'édiction de règles concernant l'activité, la mise en place d'un cursus contrôlé de formation, la création d'organisations professionnelles, la protection légale du monopole sur l'activité en question, l'établissement d'un code déontologique (Wilensky, 1964). Dans le domaine du journalisme, un modèle similaire a été proposé au même moment par Mcleod et Hawley (1964) qui concluaient à l'inachèvement du processus de professionnalisation. Le modèle linéaire de la professionnalisation est évidemment celui qu'adoptent spontanément les membres des organisations de journalistes, lorsqu'ils revendiquent des avancées en matière de contrôle sur la formation ou de déontologie. C'est aussi celui que véhiculent, implicitement, de nombreuses études qui mettent en avant 《l'inachèvement 》 de la profession (Mathien, 1998) ou, par un effet de miroir, son « éclatement» actuel qui contrasterait avec une plus grande régulation dans le passé (Charon, 1992).

2 Ce sont essentiellement des historiens qui ont travaillé sur cette question en France. Le respect de la chronologie historique comme la focalisation sur les sources d'archives émanant des organisations et syndicats de journalistes les ont conduits à adopter le modèle linéaire évoqué plus haut. Cf. par exemple Delporte (1999). On consultera aussi le travail d'un journaliste historien (Ferenczi, 1993).

3 On sait que le passage par les cursus reconnus par la profession ne concerne qu'une petite partie des nouveaux titulaires de la carte de journaliste professionnel chaque année en France. Cf. aussi la thèse récente de Ivan Chupin (2008) qui montre les difficultés du système de formation au journalisme à participer à la régulation de la profession. Dans le cas britannique, proche en cela du cas français, les journalistes n'ont que très marginalement limité l'accès à leur marché du travail en recherchant une forme de certification par l'État ou en contrôlant les filières de formation au métier (Aldridge \& Evetts, 2003).
} 
» professionnel, à savoir un corpus de règles collectives que se donnerait le groupe professionnel des journalistes, et qu'il s'em- ploierait à faire respecter.

C'est dans le domaine de la déontologie que l'absence de ce « code » est la plus frappante. Le sujet est abondamment commenté, notamment depuis les années 1990. Il est impossible

d'en rendre compte ici dans le détail. ${ }^{4}$ Cependant, il est assez aisé de constater que, pour l'heure, l'absence d'un véritable code déontologique propre aux journalistes a conduit de nombreux acteurs à intervenir dans ce domaine, comme les pouvoirs publics ou certains groupes d'intérêt. C'est surtout la justice qui a joué ce rôle de façon très marquée dans la période récente. Comme le constatait un avocat en 1993, l'absence d'un code prescriptif à l'intérieur de la profession a en effet conduit le code judiciaire à s'appliquer de plus en plus nettement dans la régulation du travail des journalistes. 5

Il est sans doute possible d'imaginer qu'un autre modèle de la professionnalisation permettrait de répondre favorablement à la question posée dans la première ligne de cet article. 6 Cependant, c'est plus souvent la pertinence même du modèle fonctionnaliste des professions, qui a été remise en cause. Pour le cas français à nouveau, l'absence d'un « code » journalistique bien défini a conduit à caractériser le journalisme comme un 《 professionnalisme du flou » (Ruellan, 1993), une notion qui renvoie davantage au positionnement rhétorique du groupe des journalistes (le flou étant utile à la défense du territoire des acteurs) qu'à sa construction réelle. Cyril Lemieux (2000) a, quant à lui, proposé de voir dans le journalisme une profession " postmoderne ». Inapte à la codification de ses pratiques, elle place ses membres dans l'obligation de justifier leurs actes en permanence.

Dans cet article on essaiera de montrer que la faiblesse de ce « code » professionnel ne doit pas faire conclure à l'absence d'une activité de réglage des pratiques des journalistes sur des normes professionnelles. Au contraire, l'hypothèse que nous défendrons est la suivante : au lieu que ces normes soient rassemblées dans un code exogène à l'activité elle-même, et qui s'imposerait à tous par la force des institutions qui en assurent la garde, elles sont dispersées dans l'activité quotidienne des journalistes et utilisées de façon stratégique pour défendre des conceptions concurrentes de ce métier. On substituera donc à la question de savoir si les journalistes connaissent les « codes» de leur profession, celle de savoir comment ils tentent de produire, dans la matière même de leur activité, des types de " codage » leur permettant de revendiquer une position dans la division du travail de l'information ou d'en acquérir une nouvelle.

Afin de démontrer la pertinence de cette hypothèse, nous expliciterons dans un premier temps les notions de « codage » et de « disputes juridictionnelles » en faisant référence au

\footnotetext{
4 Pour une vision d'ensemble, cf. Charon (2000).

${ }^{5}$ Pour un point de vue assez ironique illustrant bien la tentation que peut éprouver le juriste de superposer son propre code (en l'occurrence le code de procédure pénale) sur le code journalistique défaillant, cf. Supiot (1990).

${ }^{6}$ Des tentatives de ce genre ont été menées pour montrer par exemple que si la définition d'une profession prenait davantage en compte le service «sacré » rendu par le groupe à la société, les journalistes pourraient entrer dans le champ de ce concept (Broadson, 1994) ou encore que, malgré un désintérêt manifeste pour leurs organisations professionnelles, les journalistes se reconnaissent dans des valeurs qui sont proches de celles des autres membres de groupes professionnels (Johnstone, 1976). S. Splichal et C. Sparks (1994) ont, quant à eux, proposé que le seul critère par lequel on pourrait encore rapprocher les journalistes du modèle professionnel, serait leur prétention à former une profession.
} 
modèle général des mondes sociaux, qui sert de base à l'argumentation développée dans cet article. Dans un second temps, ces notions seront mises à l'épreuve d'une étude de cas, portant sur une dispute ayant agité, à la fin des années 1990, le monde de l'information européenne à Bruxelles.

\section{MONDES / CODAGES / DISPUTES}

Le modèle que nous présentons dans cet article se rattache à la perspective en termes de mondes sociaux définie par Anselm Strauss (1992) dans une allocution fameuse. Cette perspective permet d'articuler trois niveaux d'analyse trop souvent disjoints : l'analyse des activités situées dans le monde étudié et l'observation du travail des acteurs qui y évoluent ; celle des groupes sociaux qui se rencontrent dans ces mondes et de leurs rapports souvent concurrents ; celle, enfin, des univers de discours qui, eux aussi, se développent dans le monde en question.

Ce modèle a, depuis, largement été utilisé pour décrire les univers dans les- quels agissent des professionnels et, plus généralement, tous les acteurs des « drames sociaux du travail »(Hughes, 1976). Dans le cas des mondes traver- sés par des acteurs qui, au-delà d'un simple métier, revendiquent une identité professionnelle, une part de l'activité de ces acteurs consiste, en effet, à définir les contours de leur travail et leur place dans la division de ce travail. Comme le dit Hughes, « les membres des professions ne se bornent pas à offrir un service, mais [ils] définissent les besoins mêmes qu'ils servent »(Hughes, 1996a, p. 131)

Les acteurs des mondes sociaux sont donc engagés dans la production de « rhétoriques professionnelles »(Chapoulie, 1973) qui leur permettent de masquer les aspects les plus matériels de leur travail, et de mettre en avant les services rendus à leurs clients ou au public ainsi que l'intérêt général. Une des caractéristiques de ces rhétoriques est, cependant, qu'elles sont produites en même temps que le travail pour lequel le professionnel est sollicité. Celuici, s'il veut maintenir sa position dans le monde social, doit en effet s'astreindre, avec les autres participants au même monde social, à un véritable « travail permanent d'entretien de la croyance » (Paradeise, 1985, p. 31). Il serait donc erroné de séparer l'ordre des discours de celui des situations et des contextes du travail quotidien. Les discours ne peuvent en effet être réduits au seul mécanisme de la légitimation ou de la justification (a posteriori) de monopoles sur des segments d'activité ; ils sont à l'œuvre dans la même chronologie que le travail, et participent à la revendication du statut autant qu'à sa légitimation ou à sa justification 8 .

Cette inscription des univers de discours dans les mondes professionnels est au cœur du modèle 《 juridictionnel » d'analyse du professionnalisme développé à la fin des années 1980 par A. Abbott (1988). Dans ce modèle, la profession est définie avant tout par sa capacité à maîtriser une « zone de travail », c'est-à-dire à construire, certes, les conditions de la clôture

\footnotetext{
${ }^{7}$ La sociologie des professions d'inspiration interactionniste a cependant souvent considéré comme secondaire ce que disent et ce qu'écrivent les professionnels dans les mondes sociaux. Suivant le conseil de Strauss lui-même, elle a le plus souvent mis en avant les « faits palpa- bles» au détriment des 《 mondes de discours 》 (Strauss, 1992, p. 272). Eliot Freidson a bien relevé ce point en notant que la définition interactionniste des groupes professionnels se joue avant tout « dans le travail» (Freidson, 1983, p. 27).

8 « Jeux d'organisation et jeux de discours sont analysés comme deux registres interdépendants d'un travail social de construction et de stabilisation des interactions qui aident une occupation à conquérir et pérenniser un statut. »(Paradeise, 1988, p. 12).
} 
légale de son domaine d'intervention, mais aussi un ensemble "d'archétypes » et de schèmes d'interprétation des problèmes sociaux susceptibles de lui garantir une forme d'autorité sur ces problèmes 9 . La « juridiction» qui en découle, pour reprendre le terme d'Abbott, a une dimension juridique et politique évidente dans la mesure où les groupes en question usent des arènes judiciaires et des politiques publiques pour s'imposer dans la division du travail autour du problème social qu'ils traitent (santé, libertés individuelles, information). Pour autant, elle ne s'y limite pas. D'autres arènes, dont le lieu de travail et l'opinion publique, sont au quotidien le cadre de « disputes » visant à exclure des groupes concurrents dans la division du travail, par un travail de « revendication juridictionnelle » tissant patiemment, au moyen de mots, des liens de plus en plus étroits entre une zone d'activité et un groupe susceptible de l'occuper. C'est donc en inscrivant les discours des acteurs dans la dynamique des situations de travail, que ce modèle échappe à une lecture des phénomènes rhétoriques réduisant ceux-ci au seul mécanisme de la légitimation (toujours a posteriori) de monopoles sur des segments d'activité. Ces dis- cours sont à l'œuvre dans la même chronologie que le travail, et participent à la revendication du statut autant qu'à sa légitimation. Si l'on veut comprendre le fonctionnement des mondes sociaux, il est donc nécessaire de mêler étroitement l'analyse des champs du discours et celle des champs d'activité (Larson, 1990).

Dans ce qui suit, on se propose d'appliquer ce modèle en examinant non pas les codes professionnels du journalisme et leur plus ou moins grande application dans des situations concrètes, mais l'activité permanente de « codage » discursif, dont ce métier est le lieu. Le terme de « codage », entendu comme le fait de relier en permanence une zone d'activité à celui qui l'occupe, ou encore de mettre un «nom »sur cette activité10, nous permettra de prendre en compte la réflexivité discursive des acteurs comme une forme à part entière de leur travail11.

L'usage que nous ferons de ce terme est donc en partie inspiré par l'article très célèbre de $\mathrm{S}$. Hall (1994) intitulé « Codage / décodage ». Si, à l'instar de Hall, nous essaierons de mettre en évidence des «transactions discursives » dans le processus de fabrication de l'information, c'est cependant bien du côté des professionnels, et non de l'audience, que nous nous placerons. Le processus de « codage » par les professionnels nous apparaît en effet tout aussi problématique que celui de « décodage »par l'audience. C'est donc le rapport entre code et codage qui nous intéresse particulièrement ici et pas celui entre codage et décodage. Pour le dire dans les termes de M. Foucault (1981), ce rapport met en relation un « code» qui dicte

\footnotetext{
${ }^{9}$ Pour une illustration dans un tout autre domaine, celui des politiques de sécurité routière et de la prévention de l'alcool au volant, cf. Gusfield (2008).

10 Pour E. Hughes, la profession peut, en effet, se définir comme « un travail qui porte un nom » (Hughes, 1996, p. 75).

11 Roselyne Ringoot et Jean-Michel Utard ont récemment proposé un cadre d'analyse assez proche en partant de l'idée que, ce qui caractérise le journalisme contemporain est la 《dispersion 》 de ses 《formations discursives 》 et plus généralement le renversement de la relation entre profession et discours : "Notre conviction est que le journalisme est une pratique sociale de production discursive avant d'être une profession » (Ringoot \& Utard, 2005). Plus générale- ment, la perspective développée ici doit aux notions de « paradigmes 》 (Charron \& De Bonville, 1996) et de « communautés interprétatives» (Zelizer, 1993) telles qu'elles ont été appliquées au journalisme. Il faut cependant noter que c'est bien la dispersion conflictuelle de ces «paradigmes » qui est ici au centre de l'analyse et pas leur convergence vers des formes canoniques ou conventionnelles. Lorsque l'on prend la mesure de la variabilité des pratiques professionnelles en matière journalistique, on est en effet conduit à se représenter leur rencontre sur le modèle d'un champ de lutte, dans lequel les 《stratégies discursives 》 sont extrêmement diverses et importantes (Chalaby, 1996).
} 
comment faire un certain nombre de choses, et un « discours de vérité » qui en justifie en permanence la raison.

Il ne s'agit donc pas d'une construction intellectuelle. Ce rapport est entièrement contenu et déployé dans le travail quotidien des professionnels, tous pris dans ce que le spécialiste du savoir médical appelait une "ronde » autour d'un problème social donné ${ }^{2}$. Saisir cette ronde suppose donc d'abord d'observer le travail des professionnels et d'écouter leur argumentation au moment où celle- ci se forme. Plutôt que dans l'entretien rétrospectif, c'est donc dans l'analyse des produits de l'activité journalistique (des textes pour l'essentiel), que l'on cherchera la trace d'un « codage » professionnel. Plus que le « sens » hypothétique de ces textes, on cherchera aussi le lien qui s'établit, de manière diffuse mais répétitive, entre son auteur et le sujet qu'il traite, ce qui justifie le recours à la statistique textuelle plutôt qu'à l'analyse de contenu13. Enfin, conformément à la dimension écologique du modèle, la recherche devra porter au maximum sur des situations de controverses, dans lesquelles plusieurs types de codages sont à l'œuvre en même temps.

\section{UNE ANALYSE DE CAS : LA DISPUTE DE BRUXELLES OU LE CODAGE CONTROVERSÉ DE L'ACTUALITÉ EUROPÉENNE}

Le modèle que nous venons de définir est ici appliqué au monde de l'informa- tion européenne à Bruxelles14. De nombreuses disputes - déjà bien connues pour certaines (Baisnée, 2001 ; Bastin, 2004 ; Georgakakis, 2000)15 — ont agité ce monde à la fin des années 1990. Dans un contexte de développement du journalisme d'investigation, la crise qui mena à la démission de la Com- mission européenne dans la nuit du 15 au 16 mars 1999, s'accompagna par exemple d'une violente querelle journalistique. Deux définitions du journalisme semblent alors s'opposer parmi les journalistes présents dans la salle de presse de la Commission européenne. Les uns, considérés comme des « amis 》 par l'institution, défendaient un journalisme « de fond », et les autres, devenus « ennemis », un journalisme 《 d'investigation $\gg 16$.

12 Il s'agit par exemple, à propos du savoir médical, de la « ronde de parents, de nourrices, de domestiques, de pédagogues, de médecins », qui entoure de ses savoirs pratiques le corps de l'enfant dès sa naissance (Foucault, 1976, p. 130).

13 Ce que, là encore, on peut renvoyer à la formulation de Foucault (1969, p. 126) selon laquelle, dans les formulations tirées de la ronde mentionnée plus haut, la production du sens compte moins que la capacité pour un groupe à rester sujet de son activité : « Décrire une formulation en tant qu'énoncé ne consiste pas à analyser les rapports entre l'auteur et ce qu'il a dit (ou voulu dire sans le savoir) ; mais à déterminer quelle est la position que peut et doit occuper tout individu pour en être le sujet. »

14 Pour une présentation d'ensemble plus détaillée, cf. Bastin (2003). Les éléments présentés ici sont notamment tirés du chapitre 11.

15 Pour mémoire, la crise institutionnelle commence à l'automne 1998 avec la publication dans les médias d'une série de révélations sur les pratiques d'embauche de certains commissaires (dont la française Édith Cresson). À partir de décembre, le traitement de ce sujet devient clairement politique avec le refus par le Parlement européen de voter la décharge budgétaire à la Commission le 17 décembre, puis la nomination d'un Comité des Sages le 27 janvier. C'est la remise d'un rapport très critique par ce comité le 15 mars 1999, qui provoque la démission de l'exécutif européen.

${ }^{16}$ Les termes sont empruntés à une note du porte-parole de la commissaire Édith Cresson : « Il est faux de dire que nous n'avons plus d'amis. Au contraire : beaucoup de journalistes avouent leur perplexité devant ce qui arrive ; beaucoup désapprouvent - parfois ouvertement - l'outrance de leurs collègues [...] Nous devons utiliser nos alliés potentiels pour rétablir un équilibre entre journalistes de fond et d'investigation. 》 (« Une note secrète de la Commission européenne. Bruxelles rêve de dicter l'info », Libération, 30 janvier 1999). 
Pour suivre la controverse par le biais des stratégies discursives de ses acteurs et afin d'entrer dans la matière même des produits de ces journalistes, nous avons constitué un corpus de 225 articles écrits entre le $1^{\text {er }}$ juillet 1998 et le 30 avril 1999 par les deux professionnels français les plus engagés dans la controverse : le correspondant de Libération du côté du journalisme "d'investigation », et celui du Monde du côté du « fond »17. Ce corpus a été soumis à une analyse de classification descendante hiérarchique au moyen du logiciel Alceste 18 .

Cinq classes lexicales stables permettent de caractériser le corpus d'articles des deux journalistes. Le tableau 1 en synthétise le contenu du point de vue qui nous intéresse ici.

Tableau 1. Un espace lexical fracturé

\begin{tabular}{|c|l|r|l|l|l|}
\hline 1 & Classe & $\begin{array}{l}\text { \% du } \\
\text { corpus }\end{array}$ & Lexique caractéristique & $\begin{array}{l}\text { Mots-outils } \\
\text { caractéristiques }\end{array}$ & Journaliste associé \\
\hline $\begin{array}{l}\text { correspondance } \\
\text { diplomatique }\end{array}$ & 30,4 & $\begin{array}{l}\text { sommet, négociation, conseil, } \\
\text { agenda_2000, chef, chancelier, mars, } \\
\text { étranger, Gerhard_Schröder, } \\
\text { adhésion... }\end{array}$ & $\begin{array}{l}\text { Quinze, lors de, } \\
\text { sur, dès que, } \\
\text { avant... }\end{array}$ & $\begin{array}{l}\text { Simon }(\chi 2=72,3) \\
\text { Mathieu }(\chi 2=3,0)\end{array}$ \\
\hline 3 & $\begin{array}{l}\text { dramaturgie } \\
\text { démocratique } \\
\text { écompte-rendu }\end{array}$ & 15,5 & $\begin{array}{l}\text { censure, parlement, exécutif, motion, } \\
\text { eurodéputé, commission, socialisme, } \\
\text { démission, groupe, Santer... }\end{array}$ & $\begin{array}{l}\text { en place, hier, } \\
\text { contre, devant, } \\
\text { toutes... }\end{array}$ & Mathieu $(\chi 2=78,6)$ \\
\hline $\begin{array}{l}\text { journalisme } \\
\text { d'investigation }\end{array}$ & 14,8 & $\begin{array}{l}\text { euro, finance, monétaire, zone_euro, } \\
\text { monnaie, euro_11, banque, euroland, } \\
\text { Strauss_Kahn, économie... }\end{array}$ & $\begin{array}{l}\text { donc, et, ont, } \\
\text { nous, leurs... } \\
\text { Edith_Cresson, ami, embaucher, } \\
\text { société, Berthelot, Perry, enquête... }\end{array}$ & $\begin{array}{l}\text { Mathieu }(\chi 2=40) \\
\text { ses, parce que... }\end{array}$ & $\begin{array}{l}\text { Mathieu } \\
(\chi 2=110,8)\end{array}$ \\
\hline 5 & $\begin{array}{l}\text { pédagogie } \\
\text { agricole }\end{array}$ & 23,8 & $\begin{array}{l}\text { dépense, contribution, milliard, } \\
\text { agricole, prix, aide, viande, baisse, } \\
\text { bovin, budget... }\end{array}$ & $\begin{array}{l}\text { ils, environ, en } \\
\text { outre, pour, } \\
\text { autre... }\end{array}$ & Simon $(\chi 2=147)$ \\
\hline
\end{tabular}

La première classe peut être décrite comme celle de la correspondance diplomatique. Elle est fortement associée aux articles du journaliste de « fond ». Elle est marquée par le vocabulaire des "sommets 》 et des "chefs d'État et de gouvernement 》 qui y participent. La construction européenne y apparaît comme un processus intergouvernemental ( « négociation », 《 diplomatie »...) rythmé par un 《agenda » institutionnel fort ( «phase », «programmer »...).

17 Ce corpus comprend l'ensemble des articles écrits, pendant cette période, par ces deux correspondants que nous désignerons par des pseudonymes : Mathieu pour Libération et Simon pour Le Monde. Nous avons aussi recueilli l'ensemble des articles cosignés avec un autre journaliste (ils figurent dans l'analyse avec un « + » à côté du nom de l'auteur)

18 La méthode consiste à segmenter le texte, puis à caractériser chaque segment par son vocabulaire avant de regrouper, par le biais d'une analyse de classification, les segments pré- sentant la plus grande proximité lexicale entre eux et les plus grandes oppositions avec le reste du corpus. Une mesure de $\chi 2$ permet de connaître le lexique le plus spécifique à chacune des classes ainsi constituées, ainsi que le degré d'association de chaque classe à l'un ou l'autre des journalistes auteurs des articles. Une analyse factorielle des correspondances permet enfin de représenter le « monde lexical», dans lequel viennent se positionner l'ensemble des formes analysées ainsi que les modalités des variables d'analyse (Reinert, 1993 ; Reinert, 1998). La méthode repose donc bien sur la mise en évidence de régularités lexicales dans le corpus et la construction d'une représentation de la dispersion des formes les plus récurrentes dans des classes antagonistes. 
La deuxième classe est celle de la dramaturgie démocratique, dans laquelle le Parlement prend la première place ( " parlement », " parlementaire », " euro- parlement 》, 《eurodéputé »...). Les clivages politiques apparaissent aussi dans cette classe ( "chrétien », "vert ", " socialisme ») ainsi que les termes qui renvoient aux procédures du contrôle démocratique exercé par le Parle- ment ( "vote 》, " motion 》, " censure ", "sanctionner »). Le récit se fait précis et est mis en scène comme tel ( "hier », « nuit »...) ; l'attention est focalisée sur quelques personnages ( "Cresson 》, " Marin », "Delors »). Cette classe est clairement associée aux articles du journaliste « d'investigation ».

La troisième classe est celle du compte-rendu des décisions économiques. Les " réunions 》 ont remplacé les sommets et les "gouverneurs 》, les chefs de gouvernement. Les arènes décrites ne sont plus des institutions politiques, mais des "clubs », et l'on se trouve dans le domaine de l' « informel 》. Le vocabulaire économique est très présent ( " croissance ", 《déficit »...). Les registres d'argumentation qui correspondent à cette classe, s'éloignent très peu du simple exercice logique ( donc », « et »). Cette classe est associé au journaliste « d'investigation ».

La quatrième classe relève du journalisme d'investigation sur les affaires. Le lexique caractéristique de cette classe se compose de nombreux termes renvoyant au domaine de la "fraude » et de sa révélation. Les comportements incriminées relèvent clairement de l' " emploi » " fictif» ( " contrat », " embaucher »), et le lexique explore toutes les formes des relations de proxi- mité et de dépendance que celui-ci exploite et produit ( "proche », " charger », " payer »). Le vocabulaire de l' " enquête » ("irrégularité 》, " plainte », " épingler ») participe d'une invocation de principes de "justice »: la référence au "public » et à la responsabilité ( "répondre »). La forte présence de pronoms, notamment à la première personne, signale l'importance dans cette classe des citations faites par le journaliste de propos au style direct et des publications d'extraits d'entretiens (dans lesquels les acteurs principaux des «affaires»se justifient). On est aussi dans le registre de l'ex- plication avec "parce qu'». On voit donc se déployer là un registre lié à une pratique très 《 investigatrice » du journalisme, qui n'hésite pas à renvoyer à la certitude de faits établis, de soupçons et de valeurs morales (par exemple "fait 》, " doute 》 et "faute » font partie de cette classe). Cette classe est fortement associée au journaliste « d'investigation ».

Enfin la cinquième et dernière classe est celle de la pédagogie du contentieux agricole. Le vocabulaire de cette classe est nettement marqué par le champ sémantique " agricole » et par les domaines de politique européenne liés à ce secteur ( $P A C »)$. Si les termes associés à une lecture diplomatique de cette politique sont nombreux ( "Paris », "Irlande »...), ainsi que ceux qui dénotent le contentieux qui l'entoure ("compenser 》, "autoriser 》, " interdiction »...), on peut noter aussi une forte insistance mise sur l'explicitation des " mécanismes » de cette politique (par exemple le recours aux sigles : "PAC», "PIB», « $O M C$ » ou encore les termes techniques comme "progressif 》, "stabiliser ", "prix »). Les opérateurs de traduction du registre technique sont nombreux : " environ 》, " c'est-àdire », " de manière ». Cette classe est très clairement associée au journaliste de " fond ».

La cohérence des classes produites est d'abord un signe de la routinisation du travail des deux correspondants. Elle signale aussi l'importance prescriptive de l'agenda institutionnel 
sur ce travail19. On peut par exemple constater que près de la moitié du corpus s'inscrit dans les classes 1 et 3 . On atteint même $70 \%$ en ajoutant la classe 5 . Or ces trois classes sont celles dont le vocabulaire est le plus technique et la posture la plus neutre. La source institutionnelle possède donc à l'évidence, dans ce monde de l'information, un important pouvoir de définition première des sujets faisant l'agenda médiatique.

Il n'en reste pas moins que le monde lexical produit par les deux journalistes est fracturé. Le traitement des « affaires » s'y oppose très fortement au traitement des sujets diplomatiques, économiques et agricoles. Cette ligne de fracture lexicale correspond par ailleurs à une opposition entre les deux journalistes. La routine narrative représentée par les classes 1, 3 et 5 (à laquelle participent d'ailleurs les deux journalistes) est en effet concurrencée, dans les textes produits par ces journalistes, par deux nouvelles classes lexicales (les classes 2 et 4), fortement associées au journaliste de Libération, dont le vocabulaire est très spécifique. Cette fracture apparait nettement sur la projection factorielle du nuage lexical (cf. graphique $1)^{20}$. Le premier axe structurant de ce nuage représente près de $35 \%$ de sa variance totale. Il oppose nettement le lexique de l'investigation (à gauche) à celui de l'analyse de fond (à droite).

Mais ce sont aussi deux façons d'articuler les mots de la réalité décrite dans les articles des deux journalistes, qui apparaissent sur ce graphique. L'outillage argumentatif oppose, en effet, assez nettement du discours (à gauche) au récit (à droite). Le discours, caractéristique $\mathrm{du}$ 《 codage » propre au journaliste « d'investigation », se caractérise par une importante intervention du locuteur dans le texte, au moyen de déictiques, c'est-à-dire de termes qui tirent tout leur sens de la situation d'énonciation (pronoms de la première et deuxième personnes, adjectifs et possessifs qui s'y rapportent, démonstratifs, adverbes de localisation spatiale et temporelle, etc.).

\footnotetext{
19 Pour mémoire : maintien d'une activité intergouvernementale forte, notamment à l'occasion des sommets européens de Pörtschach (24 et 25 octobre 1998) et Berlin (24 et 25 mars 1999), où sont âprement discutés l'agenda 2000 (négociation sur le budget de l'Union) et le financement de la PAC ; montée en puissance des nouvelles institutions économiques liées à la Monnaie unique (la valeur définitive de l'Euro est connue le 31 décembre 1998), notamment dans le contexte du conflit balkanique et de l'aide économique apportée par l'UE ; discussions agricoles sur les contributions des pays membres à la PAC et sur des sujets sanitaires (le 23 novembre est décidée la levée de l'embargo sur la viande de bœuf britannique) ou commerciaux (conflits d'intérêts avec les États-Unis à l'OMC).

20 L'analyse factorielle a été réalisée en retenant l'ensemble des items (mots-pleins, mots- outils et modalités de variables) dont la contribution à l'un des deux premiers axes ayant la plus forte inertie est supérieure à 0,003 . Ce critère permet de sélectionner 191 items. Parmi ceux-ci, les modalités de variables n'ont été projetées dans le plan factoriel que pour ce qui concerne l'identité de l'auteur des articles. Les autres (page, journal et mois) n'ont pas été utilisées (dans tous les cas, les modalités de variables sont projetées en supplémentaire, et ne participent donc pas à la détermination des axes). Cette technique ne permettant de projeter dans le plan factoriel qu'un petit nombre de mots-outils (ceux-ci ont par construction une contribution faible aux axes), j'ai ajouté sur le graphique les 38 mots-outils les plus contributifs sur l'un des deux axes, quelque soit le niveau de cette contribution. Par ailleurs, quelques mots-pleins peu contributifs mais significatifs à l'égard de la question posée ici ont, eux aussi, été projetés. Il s'agit de «faits» (au pluriel le terme désigne « les faits», et ne peut pas être confondu avec l'usage singulier, comme dans certaines locutions comme « de fait », 《 en fait »...), 《 information », 《Libération », 《 doute », 《faute », 《 presse », 《 opinion ». D'autres termes particulièrement intéressants comme 《 journaliste » ou « article » sont assez contributifs pour apparaître d'eux- mêmes sur le graphique. Les motsoutils apparaissent en gras, italique et grisé sur le graphique ; les noms des auteurs sont surlignés et gras ; les mots de la revendication juridictionnelle sont soulignés. Sur le graphique, le « journalisme d'investigation » occupe le quart Nord-Ouest, la « pédagogie agricole » le quart Nord-Est, le 《 compte-rendu économique » et la « correspondance diplomatique » le quart Sud-Est, la « dramaturgie démocratique » le quart Sud-Ouest.
} 
Enfin, l'analyse lexicale révèle l'apparition, dans la classe 4 notamment, d'un ensemble de mots qui ne désignent pas les «affaires» ou l'actualité européenne, mais le métier de journaliste. Le traitement des « affaires » s'accompagne, en effet, dans cette classe, de références explicites au lexique des activités de presse comme "journaliste ", " média ", " article » et "journal ». De même, on trouve dans cette classe le titre de la publication dans laquelle écrit le journaliste en question ( «Libération »). Ce résultat peut paraître surprenant a priori. Il signale que dans cette classe, à la différence de toutes les autres, les conditions dans lesquelles les mots sont produits, ne sont pas cachées mais exhibées. La description des faits est complétée de références à ceux qui les décrivent, et le journaliste se met en scène comme un acteur de ce qu'il écrit21.

Graphique 1. Le monde lexical de l'information européenne à Bruxelles en 1998-1999 (Analyse factorielle des correspondances)

\footnotetext{
${ }^{21}$ Le terme « journaliste », absent des articles de Mathieu avant 1997, est utilisé, au singulier ou au pluriel, au total 46 fois par le journaliste « d'investigation» (et trois fois sous la forme de l'adjectif « journalistique »). Le journaliste « de fond » ne l'utilise, quant à lui, que quatre fois (et dans un cas il s'agit de propos rapportés lors d'un entretien). Ce terme est très clairement utilisé par le journaliste d'investigation pour créer, dans ses articles, une figure professionnelle, celle du journaliste d'investigation. Celle-ci est mise en scène dans le récit des événements (je souligne): « À 18h30, les exemplaires sont distribués à la presse, dans une cohue indescriptible. Tous les journalistes sont frappés par la dureté du ton et des accusations portées par le rapport, notamment contre Cresson » (17 mars) ; " "J'exclus de démissionner." Édith Cresson l'a répété avec force, une nouvelle fois, hier, devant quelques journalistes » $(4 \mathrm{mars})$; «Interloqués par la violence de la réaction, plusieurs journalistes en poste à Bruxelles se mettent à enquêter sur cette affaire » (16 mars).
} 


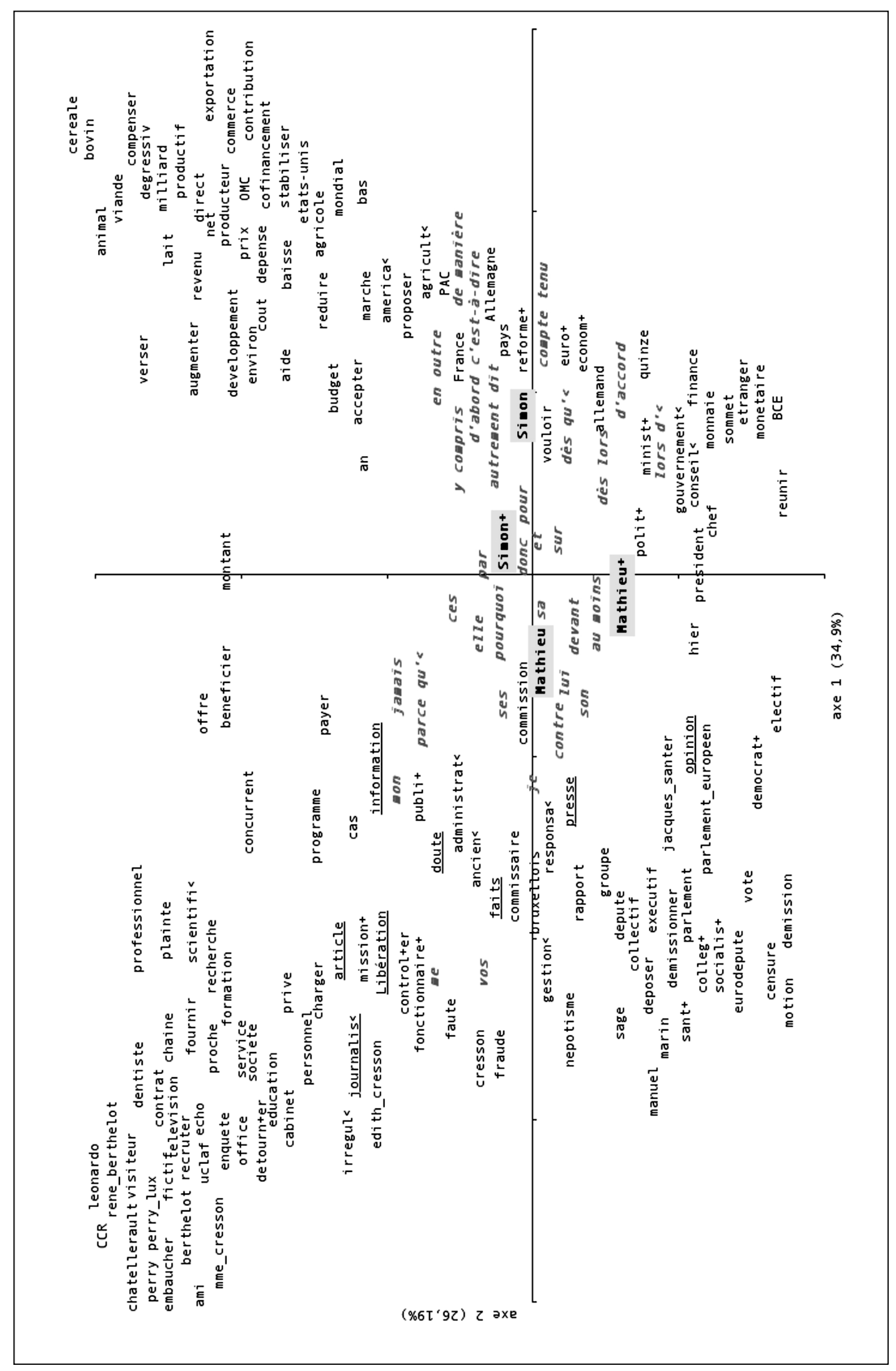


Comme l'ont montré les linguistes, les locuteurs (dans un monde de l'information, comme dans la vie courante) sont en concurrence pour des usages «scénographiés » de la langue, c'est-à-dire pour associer en permanence un discours et une situation d'énonciation, ou pour le dire autrement, un monde lexical (qu'ils peuplent de mots) et un monde social (qu'ils parcourent professionnellement)22. Ce codage fait $\mathrm{du}$ journaliste d'investigation un représentant de l'opinion publique et un garant, par son intégrité professionnelle, de la révélation de la vérité. C'est donc en réussissant à " attacher » les mots de la fraude aux mots du journalisme, que le journaliste d'investigation remet en cause de la façon la plus forte le codage professionnel dominant de l'information européenne. 23

\section{CONCLUSION}

La revendication juridictionnelle, dont on vient de voir la trace, ne se limite évidemment pas à la stratégie lexicale diffuse que nous avons appelée « codage ». Dans plusieurs articles, le journaliste d'investigation donne à cette revendication un tour plus explicite qui peut même s'apparenter à une véritable leçon sur des codes professionnels. C'est le cas par exemple d'une série d'articles, qu'il publie au moment de la dispute sur les pratiques, selon lui répréhensibles, de certains de ses collègues en matière de relation aux sources d'information ${ }^{24}$.

L'arène judiciaire elle-même fut aussi investie par cette dispute. Le journaliste d'investigation eut, en effet, à faire face, au moment de la crise évoquée plus haut, à un procès en diffamation intenté contre lui par l'attachée de presse d'Édith Cresson. Celle-ci, entrant d'une certaine façon à son tour dans la querelle sur le «nom » du métier d'informer, lui reprochait alors de l'avoir qualifiée d' « ex-journaliste » dans un article où il marquait à nouveau son territoire personnel en repoussant l'attachée de presse hors de celui-ci. Dans le droit de réponse envoyé au journal, celle-ci tenta d'opposer un autre codage du nom de journaliste, et dénonça ce qui lui apparaissait comme un déni d'identité professionnelle, une véritable atteinte à son « honneur professionnel $» 25$.

Le simple fait que ce procès ait eu lieu montre bien l'importance, pour les journalistes, de maintenir leur activité de codage et de se battre, sur de nombreux fronts, contre un codage concurrent. En l'absence d'un « code» définissant clairement les critères permettant

22 Sur cet aspect, cf. Bastin (2003, chap. 11) et Bastin (2008). Le clivage entre journalistes 《 de fond » et « d'investigation » correspond en effet aussi à un clivage entre un journaliste très engagé dans une carrière locale lui permettant de combiner de multiples engagements dans le monde bruxellois et un journaliste moins engagé localement.

23 Celui-ci repose, comme on a pu le montrer par ailleurs, sur la coproduction de l'information (Bastin, 2005).

24 Cette posture apparaît très nettement dans un article du 6 février 1999 ( (Commission européenne et journalistes : des relations ambiguës. Des voyages toute presse payée »). L'article prend prétexte d'un voyage de presse organisé par la Commission européenne en Afrique, auquel participent une trentaine de journalistes, pour dénoncer plus généralement le recours de l'institution aux professionnels de l'information pour certaines tâches de communication. Ce journaliste a par ailleurs consacré toute une série d'autres articles entre 1995 et 2001 à la définition d'une espèce de code du bon journalisme européen.

25 Elle précise : «Personne, aucune instance non plus, ne conteste la "réalité" de mon travail qui se nourrit de trente ans de carrière journalistique, dont près de dix ans comme rédactrice en chef du Nouvel Observateur, puis comme directrice adjointe de L'Express. » (Libération, 3 octobre 1998). Et comme pour mettre sa parole en actes, elle fait même, à son tour, au journaliste d'investigation une leçon de morale professionnelle, en lui reprochant de ne pas l'avoir contactée avant d'écrire son papier, « comme l'exige la déontologie de notre profession ». (Ibidem). 
d'attribuer la qualité de journaliste, c'est en effet au juge qu'il est revenu de définir le sens $\mathrm{du}$ mot « journaliste ». Le procès révéla deux définitions antagonistes du terme, deux « codages » différents de la profession. Pour le journaliste d'investigation, le journaliste est celui qui fait durablement et continuellement carrière dans ce domaine; pour la chargée de communication ayant exercé ce métier, est journaliste celui qui en a épousé la vocation, quand bien même il s'en serait éloigné pour un temps ${ }^{26}$.

Dans le tissu des mots qu'il produit au quotidien sur son terrain, le journaliste d'investigation que nous avons observé ici, a donc placé un fil très visible et très " solide », au sens où il n'hésite pas à le défendre en justice : celui des mots du journalisme. Ceux-ci construisent des équivalences discrètes entre une zone d'activité, l'information européenne, et celui qui en revendique l'occupation, le journaliste. Un « codage » professionnel est donc à l'œuvre : il tente d'imposer, pour reprendre à nouveau le vocabulaire des linguistes, " l'univers de sens qu'il institue à travers son énonciation même. » (Maingueneau, 1994, p. 276).

Ce journaliste mène de front deux entreprises. D'un côté, l'entreprise de morale, qui le conduit à mettre en circulation dans le monde de l'information européenne des mots nouveaux et controversés ( « ripoux »...). De l'autre l'entreprise de morale professionnelle, qui le conduit à y introduire les mots de la profession ( « journaliste », « presse », « information ») et à entrer en conflit avec ceux qui revendiquent aussi ces mots, ou les utilisent différemment. Ces deux entreprises sont intimement liées. Leurs mots apparaissent au même moment dans ses articles. Ils révèlent le travail patient d'un « codage » professionnel diffus, antagoniste de celui alors dominant dans le monde de l'information européenne à Bruxelles.

À travers les notions de « code » et de « codage »c'est la nature fragmentée et conflictuelle des ordres du travail journalistique, que nous avons essayée d'illustrer. À l'évidence, les acteurs des mondes de l'information ne choisissent pas à l'identique leurs pratiques, leurs carrières et les mots qu'ils emploient. Les régimes de " codage », qui permettent de lier ces niveaux différents du travail journalistique, sont de ce fait en lutte pour la maitrise des produits de ce monde. Cette lutte, toujours incertaine27, repose autant sur le contrôle des formes matérielles du monde dans lequel s'engagent les journalistes, que sur l'élaboration et l'entretien d'un codage rhétorique permettant d'asseoir une forme ou l'autre de division du travail de l'information. Les professionnels de l'information doivent, en effet, toujours choisir à la fois la carrière qu'ils font, les sources qu'ils choisissent, les codes auxquels ils adhèrent et les mots qu'ils emploient. La maîtrise de leur « juridiction » dépend de leur capacité à contrôler en même temps toutes ces dimensions de leur activité quotidienne.

\footnotetext{
26 Tribunal de grande instance de Paris, 1ère Chambre 1ère Section, jugement rendu le 26 mai 1999, $n^{\circ}$ du Rôle général : 98/24 696, page huitième.

27 Elle demande, en effet, beaucoup d'efforts au journaliste d'investigation qui se répète par exemple beaucoup dans ses articles. Comme le note Abbott (1988), plus la lecture que l'on cherche à imposer d'un problème social est éloignée des caractéristiques objectives de ce problème, plus le travail juridictionnel est lourd. Cette tâche impose d'autre part des jeux assez profonds avec l'identité professionnelle. Mathieu publie par exemple 4 points de vue dans les pages « Rebonds » de son journal en 1999 contre zéro en 1995, deux en 1996, deux en 1997, un en 1998, un en 2000 . Ces pages sont normalement réservées à des points de vue extérieurs au journal.
} 


\section{RÉFÉRENCES}

ABBOTT A. (1988), The System of Professions: an Essay on the Division of Expert Labor, Chicago, University of Chicago Press.

ALDRIDGE M. \& EVETTS J. (2003), « Rethinking the concept of professionalism: the case of journalism », The British Journal of Sociology, 54(4), p. 547-564.

BAISNÉE O. (2001), « Les relations entre la Commission et le Corps de presse accrédité auprès de l'Union européenne: crise et renouvellement des pratiques », Pôle Sud, n 15, p. $47-60$.

BASTIN G. (2003), Les Professionnels de l'information européenne à Bruxelles. Sociologie d'un monde de l'information (territoires, carrières, dispositifs), Cachan, École normale supérieure.

BASTIN G. (2004), « Les deux visages du journalisme européen. Quelques remarques depuis le monde de l'information européenne à Bruxelles », Médiamorphoses, $\mathrm{n}^{\circ} 12$.

BASTIN G. (2005), « Comment les mots viennent à Margot Wallström ? Problèmes d'intermédiation de l'action publique européenne à Bruxelles dans les années $2000 »$, G. de Terssac et D. Filâtre, (éd.), Les Dynamiques intermédiaires au coeur de l'action publique, Toulouse, Octarès.

BASTIN G. (2008), "Comment on fait un "quote". Coproduction et revendications de juridiction dans les mondes de l'information », J.-B. Legavre et P. Dauvin (dir.), Les Publics des journalistes, La Dispute, p. 65-83.

BECKER H. (1960), "Notes on the concept of commitment 》, American Journal of Sociology, 66(1), p. 32-40.

BENVENISTE E. (1966), Problèmes de linguistique générale, Paris, Gallimard.

BROADSON T. (1994), "The Sacred Side of Professional Journalism », European Journal of Communication, 9(3), p. 227-248.

CHALABY J. K. (1996), « Beyond the prison-house of language: discourse as a sociological concept », The British Journal of Sociology, 47(4), p. 684-698.

CHAPOULIE J.-M. (1973), « Sur l'analyse sociologique des groupes professionnels », Revue française de sociologie, XIV, p. 86-114.

CHARON J.-M. (1992), « Journalisme: l'éclatement », Réseaux, n 52, p. 97-113.

CHARON J.-M. (2000), "Journalisme, le défi de l'autorégulation », Réseaux, $\mathrm{n}^{\circ}$ 100, p. 385-402.

CHARRON J. \& DE BONVILLE J. (1996), « Le paradigme du journalisme de communication : essai de définition $»$, Communication, 17(2), p. 51-97.

CHUPIN I. (2008), « Les écoles du journalisme. Les enjeux de la scolarisation d'une profession (1899-2008) », Thèse de science politique (dir. B. Gaïti), Université Paris Dauphine, décembre 2008.

DELPORTE C. (1999), Les Journalistes en France 1880-1950. Naissance et construction d'une profession, Paris, Le Seuil. 
DIRECTION DU DÉVELOPPEMENT DES MÉDIAS \& CRAP (2001), Devenir journalistes. Sociologie de l'entrée sur le marché du travail, Paris, La Documentation française.

DUBAR C. \& TRIPIER P. (1998), Sociologie des professions, Paris, Armand Colin.

FERENCZI T. (1993), L'Invention du journalisme en France. Naissance de la presse moderne à la fin du XIX ${ }^{e}$ siècle, Paris, Plon.

FOUCAULT M. (1969), L'Archéologie du savoir, Paris, Gallimard.

FOUCAULT M. (1976), Histoire de la sexualité 1. La volonté de savoir, Paris, Gallimard.

FOUCAULT M. (1981), L'Ordre du discours, Paris, Gallimard.

FREIDSON E. (1983), "The theory of the professions: the state of the art », R. Dingwall and P. Lewis (ed.), The Sociology of the Professions, London, Macmillan.

GEORGAKAKIS D. (2000), "La démission de la Commission européenne: scandale et tournant institutionnel (octobre 1998-mars 1999) », Cultures et Conflits, n 38-39.

GUSFIELD J. (2008), La Culture des problèmes publics. L'alcool au volant : la production d'un ordre symbolique, Paris, Economica.

HALL S. (1978), Policing the Crisis. Mugging, the State, and Law and Order, London, Macmillan.

HALL S. (1994), « Codage/Décodage », Réseaux, n 68, p. 29-39.

HUGHES E. C. (1976), "The Social Drama of Work », Mid-American Review of Sociology, 1(1), p. 1-7.

HUGHES E. C. (1996a), « Métiers modestes et professions prétentieuses: l'étude comparative des métiers », Le Regard sociologique: essais choisis, Paris, EHESS.

HUGHES E. C. (1996b), « Le travail et le soi », Le Regard sociologique: essais choisis, Paris, EHESS.

JOHNSTONE J. (1976), The News People, University of Illinois Press.

LARSON M. S. (1990), « On the matter of experts and professional, or how impossible it is to leave nothing unsaid », R. Torstendahl and M. Burrage (ed.), The Formation of the Professions. Knowledge, State and Strategy, London/New Delhi, Newbury Park/Sage.

LEMIEUX C. (2000), Mauvaise Presse. Une sociologie compréhensive du travail journalistique et de ses critiques, Paris, Métailié.

LETEINTURIER C. (2003), « L'hétérogénéité des journalistes », Hermès, n 35, p. 35-48.

MAINGUENEAU D. (1994), "Argumentation et analyse du discours (Réflexions à partir de la seconde Provinciale) », Année sociologique, n 44, p. 263-279.

MARCHETTI D. (2003), "Les ajustements du marché scolaire au marché du travail journalistique », Hermès, n 35 , p. 81-89.

MATHIEN M. (1998), « Le journalisme professionnel face aux mutations de l'information. Déroute des valeurs ou réaction ? », Quaderni, $\mathrm{n}^{\circ} 37$. 
MCLEOD J.-M. and HAWLEY S. E. (1964), " Professionalization among newsmen 》, Journalism Quarterly, ${ }^{\circ}$ 41, p. 529-539.

PARADEISE C. (1985), « Rhétorique professionnelle et expertise », Sociologie du travail, $27(1)$, p. $17-31$.

PELISSIER N. \& RUELLAN D. (2003), " Les journalistes contre leur formation ? 》, Hermès, n 35 , p. 91-98.

REINERT M. (1993), « Les "mondes lexicaux" et leur "logique" à travers l'analyse statistique d'un corpus de récits de cauchemars », Langage et société, $\mathrm{n}^{\circ}$ 64, p. 5-39.

REINERT M. (1998), « Mondes lexicaux et Topoï dans l'approche Alceste », Mots chiffrés et déchiffrés. Mélanges offerts à E. Brunet, Honoré Champion.

RINGOOT R. and UTARD J.-M. (2005), « Genres journalistiques et "dispersion" du journalisme », R. Ringoot and J.-M. Utard (éd.) Le Journalisme en invention. Nouvel- les pratiques, nouveaux acteurs, Rennes, Presses universitaires de Rennes.

RUELLAN D. (1993), Le Professionnalisme du flou, Grenoble, Presses universitaires de Grenoble.

SALES C. (1998), La Formation des journalistes : rapport à Mme Catherine Trautmann, ministre de la culture et de la communication, porte-parole du gouvernement, La Documentation française.

SPLICHAL S. \& SPARKS C. (1994), Journalists for the 21st Century, Norwood, Ablex Publishing Corporation.

STRAUSS A. (1992), « Une perspective en termes de monde social », I. BASZANGER (éd.), La Trame de la négociation. Sociologie qualitative et interactionnisme, Paris, L'Harmattan.

SUPIOT A. (1990), « Le jeu de miroirs du droit et des médias », Droit et sociétés, n 16, p. 313-321.

WILENSKY H. (1964), "The professionalization of everyone? », American Journal of Sociology, n 2, p. 137-158.

ZELIZER B. (1993), « Journalists as interpretative communities », Critical Studies in Mass Communication, $\mathrm{n}^{\circ} 10$, p. 219-237. 